\title{
A CONDITION OF UNIFORM EXPONENTIAL STABILITY FOR SEMIGROUPS
}

\author{
CONSTANTin Buşe AND CONSTANTIN P. Niculescu
}

Abstract. The aim of this paper is to prove that the uniform exponential stability of a strongly continuous semigroup $\{T(t)\}_{t} \geqslant 0$ (acting on a complex Hilbert space $H$ ) can be derived as a consequence of the well behavior of its numerical range in a suitable Orlicz space. More precisely, assuming that there exists an Orlicz space $E=\left(L^{\Phi}, \rho^{\Phi}\right)$ over $\mathbf{R}_{+}$such that

$$
\liminf _{\alpha \downarrow 0}\left[\alpha\left\|\exp _{-\alpha}\right\|_{E^{\star}}\right]=0
$$

and

$$
\sup _{\|x\| \leqslant 1} \rho^{\Phi}(|\langle T(\cdot) x, x\rangle|) \leqslant M<\infty
$$

then the uniform growth bound $\omega_{0}$ of the semigroup verifies an estimate of the form

$$
\omega_{0} \leqslant M_{\beta}:=\beta-\left(2 M\left\|\exp _{-\beta}\right\|_{E^{\star}}\right)^{-1}<0
$$

for some positive number $\beta$. As an application, the well posedness of an abstract infinite time Cauchy problem is discussed.

Mathematics subject classification (2000): 47D02, 47D06, 35B35.

Key words and phrases: Strongly continuous semigroup, Orlicz space, uniform exponential stability.

\section{REFERENCES}

[1] C. Bennett And S. Sharpley, Interpolation of Operators, Academic Press, Boston, 1988.

[2] L. GEARHART, Spectral theory for contraction semigroups on Hilbert spaces, Trans. Amer. Math. Soc. 236 (1978), 385-394.

[3] F. HUANG, Characteristic conditions for exponential stability of linear dynamical systems in Hilbert spaces, Ann. Diff. Eq., 1 (1983), 43-56.

[4] A. Yu. Karlovich, L. Malingranda, On the interpolation constant for Orlicz spaces, Proc. Amer. Math. Soc., 129 (2001), no. 9, 27827-2739 (Electronic).

[5] M. A. KrasnoselskiI, Ya.B. RuTiCKII, Convex functions and Orlicz spaces, Noordhoff Ltd., Groningen, 1961.

[6] L. Maligranda, Orlicz spaces and interpolation, Sem. Math. 5, Dep. Mat. Univ. Estadul de Campinas, Campinas SP, Brazil, 1989.

[7] J. M. A. M. VAN NEERVEN, The Asymptotic Behavior of Semigroups of Linear Operators, Birkhäuser Verlag, 1996.

[8] J. M. A. M. VAn Neerven, B. StRaub AND L. Weis, On the asymptotic behavior of a semigroup of linear operators, Indag. Math., N.S., 6(4), (1985), 453-476.

[9] A. J. PRITCHARD, J. ZABCZYK, Stability and stabilizability of infinite dimensional systems, SIAM Rev. 23 (1983), 25-52.

[10] J. PRÜss, On the spectrum of $C_{0}$-semigroups, Trans. Amer. Math. Soc. 284 (1984), 847-857.

[11] G. WeISS, Weak $L^{p}$-stability of linear semigroup on a Hilbert space implies exponential stability, J. Diff. Eqns., 76 (1988), 269-285. 
Mathematical Inequalities \& Applications www.ele-math.com mia@ele-math.com 